\title{
IMPULSE CONTROL OF MULTIDIMENSIONAL JUMP DIFFUSIONS
}

\author{
MARK H. A. DAVIS*, XIN GUO ${ }^{\dagger}$, AND GUOLIANG WU
}

\begin{abstract}
This paper studies regularity property of the value function for an infinite-horizon discounted cost impulse control problem, where the underlying controlled process is a multidimensional jump diffusion with possibly 'infinite-activity' jumps. Surprisingly, despite these jumps, we obtain the same degree of regularity as for the diffusion case, at least when the jump satisfies certain integrability conditions.
\end{abstract}

1. Introduction. This paper is concerned with regularity of the value function in an impulse control problem for an $n$-dimensional jump diffusion process $X(t)$.

In the absence of control, the stochastic process $X(t)$ is governed by the following SDE:

$$
d X(t)=\mu\left(X\left(t^{-}\right)\right) d t+\sigma\left(X\left(t^{-}\right)\right) d W(t)+\int_{\mathbb{R}^{l}} j\left(X\left(t^{-}\right), z\right) \tilde{N}(d t, d z), \quad X(0)=x .
$$

Here $W(t)$ is an $m$-dimensional Brownian motion and $N(\cdot, \cdot)$ a Poisson random measure on $\mathbb{R}^{+} \times \mathbb{R}^{l}$, with $W$ and $N$ independent. The Lévy measure $\nu(\cdot):=\mathbb{E}(N(1, \cdot))$ may be unbounded and $\widetilde{N}(d t, d z)$ is its compensated Poisson random measure with $\tilde{N}(d t, d z):=N(d t, d z)-\nu(d z) d t$. The parameters $b, \sigma, j$ satisfy appropriate conditions (see Section 2) to ensure the well-definedness of this SDE.

If an admissible control policy $V=\left(\tau_{1}, \xi_{1} ; \tau_{2}, \xi_{2} ; \ldots\right)$ is adopted, then $X(t)$ evolves as

$$
d X(t)=\mu\left(X\left(t^{-}\right)\right) d t+\sigma\left(X\left(t^{-}\right)\right) d W(t)+\int_{\mathbb{R}^{l}} j\left(X\left(t^{-}\right), z\right) \widetilde{N}(d t, d z)+\sum_{i} \delta\left(t-\tau_{i}\right) \xi_{i},
$$

where $\delta(\cdot)$ denotes the Dirac delta function. With this given control, the associated total expected cost (objective function) is

$$
J_{x}[V]:=\mathbb{E}_{x}\left(\int_{0}^{\infty} e^{-r t} f(X(t)) d t+\sum_{i=1}^{\infty} e^{-r \tau_{i}} B\left(\xi_{i}\right)\right) .
$$

The aim is to minimize the total cost over all admissible control policies, with the value function

$$
u(x)=\inf _{V} J_{x}[V] .
$$

HJB and Regularity. A heuristic derivation from the Dynamic Programming Principle shows that the value function (1.4) is associated with the following Quasi-Variational-Inequality, or HJB, by

$$
\max (\mathcal{L} u-f, u-\mathcal{M} u)=0 \quad \text { in } \mathbb{R}^{n} .
$$

where $\mathcal{M} \varphi(x)$ is the so called minimal operator such that

$$
\mathcal{M} \varphi(x)=\inf _{\xi \in \mathbb{R}^{n}}(\varphi(x+\xi)+B(\xi))
$$

and $\mathcal{L} \varphi(x)$ is the partial integro-differential operator

$$
\mathcal{L} \varphi(x)=-\operatorname{tr}\left[A \cdot D^{2} \varphi(x)\right]-\mu(x) \cdot D \varphi(x)+r \varphi(x)+\int_{\mathbb{R}^{l}}[\varphi(x+j(x, z))-\varphi(x)-j(x, z) D \varphi(x)] \nu(d z),
$$

where the matrix $A$ is given by $A=\left(a_{i j}\right)_{n \times n}=\frac{1}{2} \sigma(x) \sigma(x)^{\top}$. Most recently [44] proves rigorously that indeed the value function is a continuous solution to $(\underline{\mathrm{HJB}})$ in a viscosity sense.

\footnotetext{
*Department of Mathematics, Imperial College London, London SW7 2AZ, UK. Email address: mark.davis@imperial.ac.uk

${ }^{\dagger}$ Department of Industrial Engineering and Operations Research, University of California at Berkeley, CA 94720-1777. Email address: xinguo@ieor. berkeley.edu

${ }^{\ddagger}$ Department of Mathematics, University of California at Berkeley, CA 94720-3840. Email address: guoliang@math.berkeley.edu
} 
Nevertheless, an important question remains: under what conditions is the value function a solution to the (HJB) in a classical sense? Or, what is the degree of the smoothness (i.e. regularity property) for the value function in general? This is the focus of our paper.

Regularity property has been one of the central topics in PDEs theory [31, 18, 33. Besides its obvious and natural theoretical interest, regularity study provides useful insight for numerical approximation. Controls of the impulse type by allowing discrete state space and fixed cost proves most desirable for application purpose. See [10, 22, 21, 47, for risk management, 49, 37, for real options, 4, 29, 30, 14, 38, 40, for transaction cost in portfolio management, 26, 7] for insurance models, [35, 6] for liquidity risk, and [27, 39, 8, for optimal control of exchange rates. Meanwhile, jump diffusions such as Lévy processes, have been very popular in financial modeling. See for example [42, 28, 15, 9, 11, 50, 32.

Combined, there is a growing interest and need to analyze impulse controls on jump diffusions. Unfortunately, impulse control is among the hardest to analyze and the regularity study for the associated HJB or the value function is largely open, except for some special and degenerate cases such as singular control and optimal stopping problems, see [36, 43, 19, 2. One of the difficulty in establishing the regularity property lie in the non-linear, non-local operator $\mathcal{M} u$ in Eqn ( $\mathrm{HJB}$ ), Another difficulty is the partial integro-differential operator $\mathcal{L} \varphi(x)$ associated with the jump processes. For the special case when the controlled diffusion is without jumps, 3 established the regularity property by assuming that the control is bounded and nonnegative with additional smoothness in the cost structure. Recently, 20, applies the tricks of translating the regularity of the minimal operator in the action region into that of the PDEs in the continuation region. However, all these technique fail for a controlled jump diffusion component. The major issue is the additional partial integro-differential operator. Moreover, (possibly infinite) jumps through the boundary might potentially reduces the degree of smoothness for the value function.

Our work. This paper investigates the regularity of the value functions for the jump-diffusion models with impulse control. Building on the existence of the viscosity solution to the HJB for the value function [44] and the trick of 20] for the non-local minimal operator, we focus on the partial integro-differential operator in the continuation region. There are two distinct cases: when the jump is driven by compound Poisson process, or equivalently when the Lévy measure is finite, the analysis is fairly straightforward by the standard Schauder's estimate from PDEs, as in 20. For the most interesting case of infinite Lévy measure, the key is to combine the classical $L^{p}$ theory with the "bootstrap" argument to obtain regularity of the partial integro-differential operator. Finally, to deal with the regularity along the "free boundary", appropriate penalty function is devised. Surprisingly, despite the added possibly infinite jumps, we have here the same regularity as in the diffusion case, at least when the jump satisfies certain integrability conditions.

2. Assumptions and Notations. We first specify the exact mathematical framework for our problem. Given a filtered and complete probability space $\left(\Omega, \mathcal{F},\left(\mathcal{F}_{t}\right)_{t \geq 0}, \mathbb{P}\right)$ satisfying the usual conditions, we have a controlled jump diffusion process $X(t)$ as defined above at (1.2). An admissible impulse control $V$ consists of a sequence of stopping times $\tau_{1}, \tau_{2}, \ldots$ with respect to $\mathcal{F}_{t}$ and a corresponding sequence of $\mathbb{R}^{n}$-valued random variables $\xi_{1}, \xi_{2}, \ldots$ satisfying the conditions

$$
\left\{\begin{array}{l}
0<\tau_{1}<\tau_{2}<\cdots<\tau_{i}<\ldots \\
\tau_{i} \rightarrow \infty \text { a.s. as } i \rightarrow \infty \\
\xi_{i} \in \mathcal{F}_{\tau_{i}}, \quad \forall i \geq 1
\end{array}\right.
$$

The associated total expected cost (objective function) is given by (1.4) where $f$ is the "running cost", $B$ is the "transaction cost" and $r>0$ is the discount factor. We assume that all the randomness comes from $W$ and $N$, so that the filtration $\mathbb{F}=\left(\mathcal{F}_{t}\right)_{t \geq 0}$ is generated by $W$ and $N$.

We next specify detailed conditions on the coefficients to ensure the existence and uniqueness of (1.1), as well as the conditions on $f$ and $B$ in $₫ 2$.

Throughout this paper, we shall impose the following standing assumptions:

(A1) Lipschitz conditions on $\mu: \mathbb{R}^{n} \rightarrow \mathbb{R}^{n}, \sigma: \mathbb{R}^{n} \rightarrow \mathbb{R}^{n \times m}, j: \mathbb{R}^{n} \times \mathbb{R}^{l} \rightarrow \mathbb{R}^{n}$ : there exist constants 
$C_{\mu}, C_{\sigma}>0$ and a positive function $C_{j}(\cdot) \in L^{1} \cap L^{2}\left(\mathbb{R}^{l}, \nu\right)$ such that

$$
\left\{\begin{array}{l}
|\mu(x)-\mu(y)| \leq C_{\mu}|x-y|, \\
\|\sigma(x)-\sigma(y)\| \leq C_{\sigma}|x-y|, \\
|j(x, z)-j(y, z)| \leq C_{j}(z)|x-y|, \quad \forall x, y \in \mathbb{R}^{n}, z \in \mathbb{R}^{l} .
\end{array}\right.
$$

Assume also that

$$
j(x, \cdot) \in L^{1}\left(\mathbb{R}^{l} ; \nu\right) \text { for every } x \in \mathbb{R}^{n} .
$$

(A2) Ellipticity: There exists a constant $\lambda>0$ such that

$$
a_{i j}(x) \xi_{i} \xi_{j} \geq \lambda|\xi|^{2}, \quad \forall x, \xi \in \mathbb{R}^{n},
$$

where the matrix $A=\left(a_{i j}\right)_{n \times n}=\frac{1}{2} \sigma(x) \sigma(x)^{\mathrm{\top}}$.

(A3) Lipschitz condition on the running cost $f \geq 0$ : there exists a constant $C_{f}>0$ such that

$$
|f(x)-f(y)| \leq C_{f}|x-y|, \quad \forall x, y \in \mathbb{R}^{n} .
$$

(A4) Conditions on the transaction cost function $B: \mathbb{R}^{n} \rightarrow \mathbb{R}$ :

$$
\left\{\begin{array}{l}
\inf _{\xi \in \mathbb{R}^{n}} B(\xi)=K>0, \\
B \in C\left(\mathbb{R}^{n} \backslash\{0\}\right), \\
|B(\xi)| \rightarrow \infty, \text { as }|\xi| \rightarrow \infty, \text { and } \\
B\left(\xi_{1}\right)+B\left(\xi_{2}\right) \geq B\left(\xi_{1}+\xi_{2}\right)+K, \quad \forall \xi_{1}, \xi_{2} \in \mathbb{R}^{n} .
\end{array}\right.
$$

(A5) $r>2 C_{\mu}+C_{\sigma}^{2}+\int_{\mathbb{R}^{l}} C_{j}^{2}(z) \nu(d z)$.

Assumption (A1) ensures the existence and uniqueness of solutions to (1.1) (Cf. Theorem 9.1, Chapter VI, 23]). The condition (2.2) seems essential to our approach, in particular to establish the continuity property of the operator $I$ in Lemma 3.2 Readers are referred to [17] or 41] for a more detailed discussion of Lévy processes and jump diffusions.

In view of Assumption (A1) the following definitions for operators $L, I$ make sense.

$$
L \varphi(x)=-\operatorname{tr}\left[A \cdot D^{2} \varphi(x)\right]-\bar{\mu}(x) \cdot D \varphi(x)+r \varphi(x),
$$

where $\bar{\mu}=\mu-\int_{\mathbb{R}^{l}} j(x, z) \nu(d z)$ and, for Lipschitz continuous functions $\varphi$,

$$
I \varphi(x)=\int_{\mathbb{R}^{l}}[\varphi(x+j(x, z))-\varphi(x)] \nu(d z)
$$

We also adopt the following standard notations for function spaces:

$$
\begin{aligned}
& U C\left(\mathbb{R}^{n}\right)= \text { space of all uniformly continuous functions on } \mathbb{R}^{n}, \\
& W^{k, p}(U)= \text { space of all } L^{p} \text { functions with } \beta \text {-th weak partial } \\
& \text { derivatives belonging to } L^{p}, \forall|\beta| \leq k \\
& W_{0}^{k, p}(U)= \text { the closure, in } W^{k, p} \text {-norm, of smooth functions with compact support in } U, \\
& W_{\text {loc }}^{k, p}(U)=\left\{f \in W^{k, p}\left(U^{\prime}\right), \forall \text { compact } U^{\prime} \subset U\right\} \\
& C^{k, \alpha}(D)=\left\{f \in C^{k}(D): \sup _{\substack{x, y \in D \\
x \neq y}}\left\{\frac{\left|D^{\beta} f(x)-D^{\beta} f(y)\right|}{|x-y|^{\alpha}}\right\}<\infty, \forall|\beta| \leq k\right\}, D \text { compact. }
\end{aligned}
$$


3. Preliminary Results. We first establish some preliminary results under the assumptions (A1)(A5).

Lemma 3.1. The value function $u(\cdot)$ defined by (1.4) is Lipschitz.

Proof. Given an admissible control $V$, and two initial states $x_{1}, x_{2}$, denote by $X^{i}(t)$ the solution of (1.1). Apply Itô formula (for jump diffusions) (Theorem 5.1, Chapter II, 23]) to $Y(t)=|Z(t)|^{2}$, where $Z(t)=X^{1}(t)-X^{2}(t)$,

$$
\begin{aligned}
d Y(t)= & 2 Z(t) \cdot\left[\left(\mu\left(X^{1}(t)\right)-\mu\left(X^{2}(t)\right)\right) d t+\left(\sigma\left(X^{1}(t)\right)-\sigma\left(X^{2}(t)\right)\right) d W\right] \\
& +\left(\sigma\left(X^{1}(t)\right)-\sigma\left(X^{2}(t)\right)\right)\left(\sigma\left(X^{1}(t)\right)-\sigma\left(X^{2}(t)\right)\right)^{\top} d t \\
& -\int_{\mathbb{R}^{l}} 2\left(j\left(X^{1}(t), z\right)-j\left(X^{2}(t), z\right)\right) Z\left(t^{-}\right) \nu(d z) d t \\
& +\int_{\mathbb{R}^{l}}\left[\left(Z\left(t^{-}\right)+j\left(X^{1}(t), z\right)-j\left(X^{2}(t), z\right)\right)^{2}-\left|Z\left(t^{-}\right)\right|^{2}\right] N(d t, d z)
\end{aligned}
$$

Integrating from 0 to $t$, taking the expectation and then using Assumption (A1), we obtain

$$
\mathbb{E} Y(t)-\left(x_{1}-x_{2}\right)^{2} \leq\left(2 C_{\mu}+C_{\sigma}^{2}+\int_{\mathbb{R}^{l}} C_{j}^{2}(z) \nu(d z)\right) \int_{0}^{t} \mathbb{E} Y(s) d s,
$$

which implies that $\mathbb{E}\left|X^{1}(t)-X^{2}(t)\right| \leq e^{C t}\left|x_{1}-x_{2}\right|$ by Gronwall's inequality, where $C=2 C_{\mu}+C_{\sigma}^{2}+$ $\int_{\mathbb{R}^{l}} C_{j}^{2}(z) \nu(d z)$. Hence $J_{x_{1}}[V]-J_{x_{2}}[V] \leq C_{u}\left|x_{1}-x_{2}\right|$ by Assumptions (A3) and (A5), where

$$
C_{u}:=\frac{C_{f}}{r-\left[2 C_{\mu}+C_{\sigma}^{2}+\int_{\mathbb{R}^{l}} C_{j}^{2}(z) \nu(d z)\right]}>0 .
$$

By the arbitrariness of $V$,

$$
u\left(x_{1}\right) \leq J_{x_{1}}[V] \leq J_{x_{2}}[V]+C_{u}\left|x_{1}-x_{2}\right| \Rightarrow u\left(x_{1}\right) \leq u\left(x_{2}\right)+C_{u}\left|x_{1}-x_{2}\right| .
$$

Exchanging the roles of $x_{1}, x_{2}$ we get the desired result.

Lemma 3.2. $I u \in C\left(\mathbb{R}^{n}\right)$.

Proof. Given $x \in \mathbb{R}^{n}, u(y+j(y, z))-u(y) \rightarrow u(x+j(x, z))-u(x)$, as $y \rightarrow x$, for any $z \in \mathbb{R}^{l}$. Observe that if $|y-x|<1$,

$$
|u(y+j(y, z))-u(y)| \leq C_{u}|j(y, z)| \leq C_{u}\left(|j(x, z)|+C_{j}(z)|x-y|\right) \leq C_{u}\left(|j(x, z)|+C_{j}(z)\right),
$$

where $C_{u}$ is the Lipschitz constant of $u$. Since $j(x, \cdot)$ and $C_{j}(\cdot)$ are both $\nu$-integrable, the dominated convergence theorem yields the desired result. $\square$

For reference, we recall here Lemmas $3.3[3.5$ which were proved in 20$]$.

Lemma 3.3 (Properties of $\mathcal{M}$ ).

1. $\mathcal{M}$ is concave: for any $\varphi_{1}, \varphi_{2} \in C\left(\mathbb{R}^{n}\right)$ and $0 \leq s \leq 1$,

$$
\mathcal{M}\left(s \varphi_{1}+(1-s) \varphi_{2}\right) \geq s \mathcal{M} \varphi_{1}+(1-s) \mathcal{M} \varphi_{2} .
$$

2. $\mathcal{M}$ is increasing: for any $\varphi_{1} \leq \varphi_{2}$ everywhere,

$$
\mathcal{M} \varphi_{1} \leq \mathcal{M} \varphi_{2} .
$$

3. $\mathcal{M}$ maps $U C\left(\mathbb{R}^{n}\right)$ into $U C\left(\mathbb{R}^{n}\right)$ and maps a Lipschitz function to a Lipschitz function. In particular, $\mathcal{M u}(\cdot)$ is Lipschitz continuous.

LEMma 3.4. $u$ and $\mathcal{M} u$ defined as above satisfy $u(x) \leq \mathcal{M} u(x)$ for all $x \in \mathbb{R}^{n}$.

We define the continuation region $\mathcal{C}$ and the action region $\mathcal{A}$ as follows,

$$
\begin{aligned}
\mathcal{C} & :=\left\{x \in \mathbb{R}^{n}: u(x)<\mathcal{M} u(x)\right\}, \\
\mathcal{A} & :=\left\{x \in \mathbb{R}^{n}: u(x)=\mathcal{M} u(x)\right\} .
\end{aligned}
$$


Then, $\mathcal{C}$ is open, and we have

Lemma 3.5. Suppose $x \in \mathcal{A}$, then

(i) The set

$$
\Xi(x):=\left\{\xi \in \mathbb{R}^{n}: \mathcal{M} u(x)=u(x+\xi)+B(\xi)\right\}
$$

is nonempty, i.e., the infimum is in fact a minimum.

(ii) Moreover, for any $\xi(x) \in \Xi(x)$, we have

$$
u(x+\xi(x)) \leq \mathcal{M} u(x+\xi(x))-K,
$$

in particular,

$$
x+\xi(x) \in \mathcal{C} .
$$

4. Viscosity Solutions. There are different ways to define viscosity solutions. Let us begin with the most common one.

Definition 4.1. A function $u(\cdot) \in U C\left(\mathbb{R}^{n}\right)$ is called a viscosity subsolution (supersolution, resp.) of (HJB) if whenever $\varphi \in C^{2}\left(\mathbb{R}^{n}\right), u-\varphi$ has a global maximum (minimum, resp.) at $x_{0}$ and $u\left(x_{0}\right)=\varphi\left(x_{0}\right)$, we have

$$
\max \left\{\mathcal{L} \varphi\left(x_{0}\right)-f\left(x_{0}\right), \varphi\left(x_{0}\right)-\mathcal{M} \varphi\left(x_{0}\right)\right\} \leq 0 \quad(\geq 0 \text { resp. }) ;
$$

and $u$ is called a viscosity solution of (HJB) if it is both a subsolution and a supersolution.

Besides this standard definition of viscosity solutions, there are at least another two different (but equivalent) ones. The second way is to use semijets in stead of test functions. See, for instance, [13] and [44], for more details. For the purpose of proving our regularity results in Section 5 , we give a third definition below. The idea is that we impose "local" conditions (rather than global conditions as in Definition 4.1) on the test functions, and in the equation we only replace $u$ by the test function $\varphi$ in the "local" terms while still keep $u$ in the "nonlocal" terms. The same definition (in different notation) and the proof of equivalence can be found in 45. See also [46, 1] and [20, for a similar treatment.

Definition 4.2. A function $u(\cdot) \in U C\left(\mathbb{R}^{n}\right)$ is called a viscosity subsolution (supersolution, resp.) of $(\underline{\mathrm{HJB}})$ if whenever $\varphi \in C^{2}\left(\mathbb{R}^{n}\right), u-\varphi$ has a local maximum (minimum, resp.) at $x_{0}$ and $u\left(x_{0}\right)=\varphi\left(x_{0}\right)$, we have

$$
\max \left\{L \varphi\left(x_{0}\right)-f\left(x_{0}\right)-I u\left(x_{0}\right), u\left(x_{0}\right)-\mathcal{M} u\left(x_{0}\right)\right\} \leq 0 \quad(\geq 0 \text { resp. }) .
$$

$u$ is called a viscosity solution of (프) if it is both a subsolution and a supersolution.

THEOREM 4.3. The above two definitions of viscosity solutions are equivalent.

Proof. See [45, Proposition 5.4]. प

We now have the following basic result.

TheOrem 4.4 (41, 44]). The value function $u(\cdot)$ defined by (1.4) is a viscosity solution of (HJB).

This theorem was proved in [41, Theorem 9.8] as well as [44, Theorem 4.2]1 in the sense of our Definition 4.1. But when we prove the regularity result below, we found it more convenient to use Definition 4.2 More precisely, by Theorem 4.3 and Theorem 4.4 we can "identify" our value function $u(\cdot)$ with that of an impulse control problem of diffusion processes without jumps.

COROLlary 4.5. The value function $u(\cdot)$ is a viscosity solution of

$$
\max \{L u(x)-\tilde{f}(x), u(x)-\mathcal{M} u(x)\}=0 \quad \text { in } \mathbb{R}^{n},
$$

where $\tilde{f}(x)=f(x)+I u(x)$.

\footnotetext{
${ }^{1}$ In [44] the result was proved using an in principle smaller class of controls, the so-called 'Markov controls'. However, this restriction is unnecessary, as can be seen from the proofs of the analogous results in [48] or [25], or [51].
} 
5. Regularity of Value Function. In this section we study the smoothness of the value function $u$, starting with the special case of a finite Lévy measure.

5.1. Special Case: $\nu\left(\mathbb{R}^{l}\right)<\infty$. Let us first consider the special case in which the Lévy measure is finite, or equivalently, the jump diffusion $X(\cdot)$ is driven by a compound Poisson process. Then the operator $I$ enjoys the following nice property.

Lemma 5.1. Suppose $\nu\left(\mathbb{R}^{l}\right)<\infty$, then the operator I maps a Lipschitz function to a Lipschitz function.

Proof. Suppose $\varphi(x)$ is Lipschitz with $|\varphi(x)-\varphi(y)| \leq C_{\varphi}|x-y|$ for any $x, y \in \mathbb{R}^{n}$, then

$$
\begin{aligned}
|I \varphi(x)-I \varphi(y)| & \leq \int_{\mathbb{R}^{l}}|\varphi(x+j(x, z))-\varphi(y+j(y, z))| \nu(d z)+\int_{\mathbb{R}^{l}}|\varphi(x)-\varphi(y)| \nu(d z) \\
& \leq C_{\varphi} \int_{\mathbb{R}^{l}}[2|x-y|+|j(x, z)-j(y, z)|] \nu(d z) \\
& \leq C_{\varphi}\left(2 \nu\left(\mathbb{R}^{l}\right)+\int_{\mathbb{R}^{l}} C_{j}(z) \nu(d z)\right)|x-y| .
\end{aligned}
$$

So $I \varphi$ is Lipschitz. $\mathrm{Q}$

Corollary 4.5 and Lemma 5.1 together imply the regularity of $u$ in the continuation region.

Lemma $5.2\left(\boldsymbol{C}^{2, \alpha}\right.$-Regularity in $\left.\mathcal{C}\right)$. Assume that $\sigma \in C^{1}\left(\mathbb{R}^{n}\right)$ and $\nu\left(\mathbb{R}^{l}\right)<\infty$, then for any compact set $D \subset \mathcal{C}$, the value function $u(\cdot)$ is in the Hölder space $C^{2, \alpha}(D)$ for any $\alpha \in(0,1)$, and it is a classical solution of

$$
\mathcal{L} u-f(x)=0 \text { in } \mathcal{C} \text {. }
$$

Proof. Note that $u$ is a viscosity solution of (4.3) by Corollary 4.5 and hence a viscosity solution of $L u-\tilde{f}=0$ in $\mathcal{C}$. On the other hand, $\tilde{f} \in C^{\alpha}$ for any $\alpha<1$ by Lemma 5.1. Classical Schauder estimates imply the desired results. (See the proof of Lemma 5.4 below for a similar argument.) $\square$

Finally, an argument as in [20, §4] applies and yields the following

TheOREM 5.3 ( $\boldsymbol{W}_{\text {loc }}^{\mathbf{2}, \boldsymbol{p}}$-Regularity). Assume that $\nu\left(\mathbb{R}^{l}\right)<\infty$ and

$$
\sigma \in C^{1,1}(D) \text { for any compact set } D \subset \mathbb{R}^{n} .
$$

Then for any bounded open set $\mathcal{O} \subset \mathbb{R}^{n}$ and $p<\infty$, we have $u \in W^{2, p}(\mathcal{O})$.

5.2. More General Case: $j(x, \cdot) \in L^{1}(\nu)$. Next, we would like to remove the strong assumption that the Lévy measure $\nu$ is finite, and assume only our standing assumptions (A1)-(A5). Again, we first consider the regularity of $u$ in the continuation region $\mathcal{C}$, in which the linear elliptic PDE is satisfied. The difficulty is that we do not know $I u$ is Lipschitz or even Hölder continuous, but only continuous, by Lemma 3.2 .

We cannot apply Schauder estimates at this stage, but the $L^{p}$ estimates give the following

Lemma $5.4\left(\boldsymbol{W}_{\text {loc }}^{\mathbf{2}, \boldsymbol{p}}\right.$-Regularity in $\left.\mathcal{C}\right)$. Assume that $\sigma \in C^{1}\left(\mathbb{R}^{n}\right)$, then for any compact set $D \subset \mathcal{C}$, the value function $u(\cdot)$ is in the Sobolev space $W^{2, p}(D)$ for any $p<\infty$, and it is a strong solution 2 of

$$
\mathcal{L} u-f(x)=0 \text { in } \mathcal{C} \text {. }
$$

Proof. Denote by $\tilde{f}=f+I u$, which is continuous by Lemma 3.2 Consider in any open ball $B \subset \mathcal{C}$ the following Dirichlet problem

$$
\begin{cases}L w=\tilde{f}, & \text { in } B, \\ w=u, & \text { on } \partial B\end{cases}
$$

\footnotetext{
${ }^{2} \mathrm{~A}$ strong solution is a twice weakly differentiable function in the bounded domain that satisfies the equation almost everywhere.
} 
Classical $L^{p}$ theory (Cf. [18, Corollary 9.18]) asserts that the Dirichlet problem (5.2) has a unique strong solution $w \in W_{\text {loc }}^{2, p}(B) \cap C(\bar{B})$ for any $p<\infty$, since $\tilde{f} \in C(\bar{B})$ and the boundary data $u \in C(\partial B)$. Because $\sigma \in C^{1}(B), \bar{\mu} \in C^{0,1}(B)$ and $\tilde{f} \in C(B)$, this solution $w$ is in fact also a viscosity solution of (5.2) by [24, Theorem 2].

On the other hand, $u$ is also a viscosity solution of (5.2) by Corollary 4.5 Therefore, $w=u$ in $\bar{B}$ by classical uniqueness results of viscosity solutions to a linear elliptic PDE in a bounded domain(Cf. [13, Theorem 3.3]). Hence $u \in W_{\text {loc }}^{2, p}(B) \cap C(\bar{B})$.

Finally, any compact set $D \subset \mathcal{C}$ can be covered by finitely many balls $\left\{B_{r}\left(x_{k}\right)\right\}_{k=1}^{N}$ of radius $r<$ $\frac{1}{2} \operatorname{dist}(D, \partial \mathcal{C})$. Let $B=B_{2 r}\left(x_{k}\right) \subset \mathcal{C}$ in the above argument, then $u$ is in $W^{2, p}\left(\bar{B}_{r}\left(x_{k}\right)\right)$ for all $k$ and also in $W^{2, p}(D)$.

With more regularity of $u$ in the continuation region $\mathcal{C}$, we can use the "bootstrap argument" to obtain further regularity of $I u$ (and hence $u$ ) in $\mathcal{C}$.

TheOREm $5.5\left(\boldsymbol{C}^{2, \boldsymbol{\alpha}}\right.$-Regularity in $\left.\mathcal{C}\right)$. Assume that $\sigma \in C^{1}\left(\mathbb{R}^{n}\right)$, then for any compact set $D \subset \mathcal{C}$, the value function $u(\cdot)$ is in the Hölder space $C^{2, \alpha}(D)$ for any $\alpha \in(0,1)$, and it is a classical solution of

$$
\mathcal{L} u-f(x)=0 \text { in } \mathcal{C} \text {. }
$$

Proof. The key step in the proof is to show $I u \in C^{\alpha}(D)$ for any compact $D \subset \mathcal{C}$.

Take a compact set $D^{\prime}$ such that $D \subset D^{\prime} \subset \mathcal{C}$ and $\delta:=\operatorname{dist}\left(D, \partial D^{\prime}\right)<1$. Then by Lemma 5.2 $u \in W^{2, p}\left(D^{\prime}\right)$ for any $p<\infty$. By Sobolev imbedding, $u \in C^{1, \alpha}\left(D^{\prime}\right)$ for all $\alpha \in(0,1)$. Define the set

$$
E:=\left\{z \in \mathbb{R}^{l}:|j(x, z)|<\delta, \forall x \in D\right\} .
$$

Then for $z \in E^{c}=\mathbb{R}^{l} \backslash E$, there is $x \in D$ such that

$$
\delta \leq|j(x, z)| \leq|j(0, z)|+C_{j}(z)|x| \leq|j(0, z)|+C_{D} C_{j}(z)
$$

where $C_{D}=\max \{|x|: x \in D\}$ is a constant. So $|j(0, z)| \geq \delta / 2$ or $C_{j}(z) \geq \delta /\left(2 C_{D}\right)$ and

$$
\nu\left(E^{c}\right) \leq \frac{2}{\delta} \int_{\mathbb{R}^{l}}|j(0, z)| \nu(d z)+\frac{2 C_{D}}{\delta} \int_{\mathbb{R}^{l}} C_{j}(z) \nu(d z)<\infty .
$$

For any $x_{1}, x_{2} \in D$,

$$
\begin{aligned}
\left|I u\left(x_{1}\right)-I u\left(x_{2}\right)\right| \leq & \int_{E} \mid\left[u\left(x_{1}+j\left(x_{1}, z\right)\right)-u\left(x_{1}\right)\right]-\left[u\left(x_{2}+j\left(x_{2}, z\right)\right)-u\left(x_{2}\right)\right] \nu(d z) \\
& +\int_{E^{c}}\left|u\left(x_{1}+j\left(x_{1}, z\right)\right)-u\left(x_{2}+j\left(x_{2}, z\right)\right)\right|+\left|u\left(x_{1}\right)-u\left(x_{2}\right)\right| \nu(d z) \\
\leq & \int_{E} \int_{0}^{1}\left|D u\left(x_{1}+s j\left(x_{1}, z\right)\right) \cdot j\left(x_{1}, z\right)-D u\left(x_{2}+s j\left(x_{2}, z\right)\right) \cdot j\left(x_{2}, z\right)\right| d s \nu(d z) \\
& +\left|x_{1}-x_{2}\right| \int_{E^{c}} C_{u}\left(2+C_{j}(z)\right) \nu(d z) . \\
\leq & \int_{E} \int_{0}^{1}\left|D u\left(x_{1}+s j\left(x_{1}, z\right)\right)-D u\left(x_{2}+s j\left(x_{2}, z\right)\right)\right| \cdot\left|j\left(x_{1}, z\right)\right| d s \nu(d z) \\
& +\int_{E} \int_{0}^{1}\left|D u\left(x_{2}+s j\left(x_{2}, z\right)\right)\right| \cdot\left|j\left(x_{1}, z\right)-j\left(x_{2}, z\right)\right| d s \nu(d z) \\
& +\left|x_{1}-x_{2}\right| \int_{E^{c}} C_{u}\left(2+C_{j}(z)\right) \nu(d z) .
\end{aligned}
$$

Note that $x_{1}+s j\left(x_{1}, z\right), x_{2}+s j\left(x_{2}, z\right) \in D^{\prime}$ for all $0 \leq s \leq 1, z \in E$ and that $D u \in C^{\alpha}\left(D^{\prime}\right)$. Thus the first 
integral in (5.3) can be estimated by

$$
\begin{aligned}
& \|D u\|_{C^{\alpha}\left(D^{\prime}\right)} \int_{E}\left(\int_{0}^{1}\left|x_{1}-x_{2}+s\left(j\left(x_{1}, z\right)-j\left(x_{2}, z\right)\right)\right|^{\alpha} d s\right)\left|j\left(x_{1}, z\right)\right| \nu(d z) \\
\leq & \|D u\|_{C^{\alpha}\left(D^{\prime}\right)} \int_{E}\left|x_{1}-x_{2}\right|^{\alpha}\left(1+C_{j}(z)^{\alpha}\right)\left|j\left(x_{1}, z\right)\right| \nu(d z) \\
\leq & C_{1}\left|x_{1}-x_{2}\right|^{\alpha},
\end{aligned}
$$

for some constant $C_{1}>0$ independent of $x_{1}, x_{2}$, because by Hölder's inequality,

$$
\begin{aligned}
& \int_{E}\left(1+C_{j}(z)^{\alpha}\right)|j(x, z)| \nu(d z) \\
\leq & \int_{\mathbb{R}^{l}}\left(1+C_{j}(z)^{\alpha}\right)|j(x, z)| \mathbf{1}_{\{z:|j(x, z)|<1\}} \nu(d z) \\
\leq & \int_{\mathbb{R}^{l}}|j(x, z)| \nu(d z)+\left(\int_{\mathbb{R}^{l}} C_{j}(z)^{2} \nu(d z)\right)^{\frac{\alpha}{2}}\left(\int_{\mathbb{R}^{l}}|j(x, z)|^{\frac{2}{2-\alpha}} \mathbf{1}_{\{z:|j(x, z)|<1\}} \nu(d z)\right)^{\frac{2-\alpha}{2}} \\
\leq & \int_{\mathbb{R}^{l}}|j(x, z)| \nu(d z)+\left(\int_{\mathbb{R}^{l}} C_{j}(z)^{2} \nu(d z)\right)^{\frac{\alpha}{2}}\left(\int_{\mathbb{R}^{l}}|j(x, z)| \nu(d z)\right)^{\frac{2-\alpha}{2}},
\end{aligned}
$$

which is a continuous function in $x$ and has a maximum on $D$ independent of $x_{1}, x_{2}$.

The second term in (5.3) can be majored by $\|D u\|_{L^{\infty}(D)} \int_{\mathbb{R}^{l}} C_{j}(z) \nu(d z)\left|x_{1}-x_{2}\right|=: C_{2}\left|x_{1}-x_{2}\right|$ and the third term is majored by $C_{u}\left[2 \nu\left(E^{c}\right)+\int_{\mathbb{R}^{l}} C_{j}(z) \nu(d z)\right]\left|x_{1}-x_{2}\right|=: C_{3}\left|x_{1}-x_{2}\right|$. Thus, putting all three terms in (5.3) together,

$$
\left|I\left(x_{1}\right)-I\left(x_{2}\right)\right| \leq C_{1}\left|x_{1}-x_{2}\right|^{\alpha}+\left(C_{2}+C_{3}\right)\left|x_{1}-x_{2}\right| \leq C\left|x_{1}-x_{2}\right|^{\alpha},
$$

where $C_{1}, C_{2}, C_{3}$ and $C=C_{1}+\left(C_{2}+C_{3}\right)(\operatorname{diam} D)^{1-\alpha}$ are constants independent of $x_{1}, x_{2} \in D$. This proves that $I u \in C^{\alpha}(D)$.

Finally, we can repeat a similar argument in the proof of Lemma 5.2 This time we know $\tilde{f} \in C^{\alpha}(D)$, thus the solution of (5.2) is in fact in $C^{2, \alpha}(D)$ by Schauder estimates ([18, Theorem 6.13]). Thus, $u \in C^{2, \alpha}(D)$ for compact $D \subset \mathcal{C}$, and $u$ is a classical solution of $\mathcal{L} u-f=0$ in $\mathcal{C}$. $\mathrm{Q}$

Parallel to [20, once we have $C^{2, \alpha}$ regularity, we are able to obtain $W^{2, p}(D)$ regularity for any compact set $D \subset \mathbb{R}^{n}$.

TheOREm 5.6 ( $\boldsymbol{W}_{\text {loc }}^{\mathbf{2}, \boldsymbol{p}}$-Regularity). Assume that

$$
\sigma \in C^{1,1}(D) \text { for any compact set } D \subset \mathbb{R}^{n} .
$$

Then for any bounded open set $\mathcal{O} \subset \mathbb{R}^{n}$ and $p<\infty$, we have $u \in W^{2, p}(\mathcal{O})$. In particular, $u \in C^{1}\left(\mathbb{R}^{n}\right)$ by Sobolev embedding.

The proof is given in the next section. It follows similar lines to that of [20, Theorem 4.2], involving regularity of an associated optimal stopping problem.

6. Proof of Theorem 5.6. To study the regularity of the value function for the impulse control problem, we need to investigate the related optimal stopping problem. More precisely, we shall obtain the regularity of solutions for the HJB equation associated with this optimal stopping problem.

TheOrem 6.1. Suppose $\mathcal{O}$ is a bounded open set in $\mathbb{R}^{n}$ with smooth boundary. Assume

$$
\begin{aligned}
& a_{i j} \in C^{1,1}(\overline{\mathcal{O}}), \quad \mu_{i} \in C^{0,1}(\overline{\mathcal{O}}), \quad r>0, \quad f \in C(\overline{\mathcal{O}}), \\
& a_{i j} \xi_{i} \xi_{j} \geq c|\xi|^{2}, \forall x, \xi \in \overline{\mathcal{O}}, \text { for some } c>0, \\
& g \in C(\overline{\mathcal{O}}), \quad g \geq 0 \text { on } \partial \mathcal{O} .
\end{aligned}
$$

Assume also that there exist a sequence of functions $\left\{g^{\varepsilon}\right\}_{\varepsilon>0}$ and a constant $M>0$ satisfying

$$
\left\{\begin{array}{l}
g^{\varepsilon} \in C^{2}(\mathcal{O}) \cap C(\overline{\mathcal{O}}), \quad L g^{\varepsilon} \geq-M \text { in } \mathcal{O}, \\
g^{\varepsilon} \rightarrow g \text { uniformly in } \overline{\mathcal{O}}
\end{array}\right.
$$


If $v \in C(\overline{\mathcal{O}})$ is a viscosity solution of

$$
\begin{cases}\max \{L v-f, v-g\}=0 & \text { in } \mathcal{O}, \\ v=0 & \text { on } \partial \mathcal{O}\end{cases}
$$

then $v \in W^{2, p}(\mathcal{O})$.

Remark 1. Note that Assumption (6.4) is trivially satisfied if $g \in C^{2}(\overline{\mathcal{O}})$. However, later we will apply this theorem to $g=\mathcal{M} u$, which is not necessarily in $C^{2}(\overline{\mathcal{O}})$. In applications, $g^{\varepsilon}$ can be taken as the usual mollification of $g$, or its slight modification (which may only be in $C^{2}(\mathcal{O}) \cap C(\overline{\mathcal{O}})$ but not in $C^{2}(\overline{\mathcal{O}})$, as in Corollary 6.2 below).

As a corollary of Theorem 6.1 we obtain local $W^{2, p}(n<p<\infty)$ regularity of continuous viscosity solutions of

$$
\max \{L v-f, v-g\}=0 \text { in } \mathbb{R}^{n} .
$$

Corollary 6.2. Assume that $f \in C\left(\mathbb{R}^{n}\right), a_{i j} \in C_{\text {loc }}^{1,1}\left(\mathbb{R}^{n}\right)$, and $\mu, \sigma$ and $g$ are Lipschitz in $\mathbb{R}^{n}$. Assume also that for any bounded open set $\mathcal{O} \subset \mathbb{R}^{n}$ with smooth boundary, there are constants (maybe depending on $\mathcal{O}) c>0$ and $M$ such that (6.2) and (6.4) are satisfied.

If $v \in C\left(\mathbb{R}^{n}\right)$ is a viscosity solution of (6.6), then $v \in W^{2, p}(\mathcal{O})$ for any $\mathcal{O} \subset \mathbb{R}^{n}$ with smooth boundary and any $1 \leq p<\infty$, and hence also in $C^{1}\left(\mathbb{R}^{n}\right)$.

We defer the proofs of Theorem 6.1 and its corollary to the appendix, and focus now on proving our main theorem using the above corollary.

Proof. [Proof of Theorem [5.6 Given any bounded open set $\mathcal{O}$ with smooth boundary, we denote by $\mathcal{C}^{\prime}$ $\left(\mathcal{A}^{\prime}\right.$, resp.) the restriction of the continuation (action, resp.) region within $\mathcal{O}$. Then there exists an open ball $\mathcal{O}^{\prime} \supset \mathcal{O}$ such that for any $x \in \mathcal{O}, u(x+\xi)+B(\xi) \leq \mathcal{M} u(x)+1$ implies $x+\xi \in \mathcal{O}^{\prime}$. Because in this case,

$$
B(\xi) \leq \mathcal{M} u(x)-u(x+\xi)+1 \leq \mathcal{M} u(x)+1 \leq \sup _{\overline{\mathcal{O}}} \mathcal{M} u+1<\infty .
$$

But $B(\xi) \rightarrow \infty$ as $|\xi| \rightarrow \infty$, which implies that all such $\xi$ are bounded uniformly.

Now we define the set

$$
D:=\left\{y \in \mathcal{O}^{\prime}: u(y)<\mathcal{M} u(y)-\frac{K}{2}\right\} .
$$

Clearly, $\bar{D}$ is compact and $\bar{D} \subset \mathcal{C}$. From Lemma 5.2 ,

$$
u \in C^{2, \alpha}(\bar{D}) \text {. }
$$

For any $x \in \mathcal{O}$, take a minimizing sequence $\left\{\xi_{k}\right\}$ such that $u\left(x+\xi_{k}\right)+B\left(\xi_{k}\right) \rightarrow \mathcal{M} u(x)$. Then $\left\{\xi_{k}\right\} \subset \mathcal{O}^{\prime}$. Extract a convergent subsequence (still denoted by $\left\{\xi_{k}\right\}$ ) converging to $\xi^{*}$. Because $B(\xi)+B\left(\xi^{\prime}\right) \geq K+$ $B\left(\xi+\xi^{\prime}\right)$

$$
\begin{aligned}
\mathcal{M} u(x) & =\inf _{\eta \in \mathbb{R}^{n}}\left\{u\left(x+\xi_{k}+\eta\right)+B\left(\xi_{k}+\eta\right)\right\} \\
& \leq \inf _{\eta \in \mathbb{R}^{n}}\left\{u\left(x+\xi_{k}+\eta\right)+B(\eta)\right\}+B\left(\xi_{k}\right)-K \\
& =\mathcal{M} u\left(x+\xi_{k}\right)+B\left(\xi_{k}\right)-K \\
& =\mathcal{M} u\left(x+\xi_{k}\right)-u\left(x+\xi_{k}\right)+\left[u\left(x+\xi_{k}\right)+B\left(\xi_{k}\right)\right]-K .
\end{aligned}
$$

Passing to the limit $k \rightarrow \infty$, we obtain

$$
u\left(x+\xi^{*}\right)-\mathcal{M} u\left(x+\xi^{*}\right) \leq-K .
$$

In particular, $y:=x+\xi^{*} \in D$. 
On the other hand, since $u-\mathcal{M} u$ is uniformly continuous on $\overline{\mathcal{O}^{\prime}}$, there exists $\rho_{0}>0$ such that

$$
\left|y-y^{\prime}\right| \leq \rho_{0} \Rightarrow\left|u\left(y^{\prime}\right)-\mathcal{M} u\left(y^{\prime}\right)-(u(y)-\mathcal{M} u(y))\right| \leq \frac{K}{4} .
$$

Hence, for all $\rho \in\left(0, \rho_{0}\right], \lambda \in[-1,1]$ and unit vector $\chi \in \mathbb{R}^{n}$,

$$
y=x+\xi^{*} \in D, \quad y^{\prime}=y+\lambda \rho \chi \in D,
$$

because $u\left(y^{\prime}\right)-\mathcal{M} u\left(y^{\prime}\right) \leq u(y)-\mathcal{M} u(y)+\frac{K}{4}<-\frac{K}{2}$.

Since $\mathcal{M} u(x \pm \rho \chi) \leq u\left(x \pm \rho \chi+\xi_{k}\right)+B\left(\xi_{k}\right)$ for all $k$,

$$
\begin{aligned}
& \mathcal{M} u(x+\rho \chi)+\mathcal{M} u(x-\rho \chi)-2 \mathcal{M} u(x) \\
\leq & u\left(x+\rho \chi+\xi_{k}\right)+u\left(x-\rho \chi+\xi_{k}\right)+2 B\left(\xi_{k}\right)-2 \mathcal{M} u(x) \\
\rightarrow & u(y+\rho \chi)+u(y-\rho \chi)-2 u(y), \quad k \rightarrow \infty,
\end{aligned}
$$

and hence the second order difference quotient at $x$

$$
\begin{aligned}
& \frac{1}{\rho^{2}}[\mathcal{M} u(x+\rho \chi)+\mathcal{M} u(x-\rho \chi)-2 \mathcal{M} u(x)] \\
\leq & \frac{1}{\rho^{2}}[u(y+\rho \chi)+u(y-\rho \chi)-2 u(y)] \\
= & \frac{1}{|\rho|} \int_{0}^{1}[(D u(y+\lambda \rho \chi)-D u(y-\lambda \rho \chi)] \cdot \chi d \lambda \\
\leq & C_{D},
\end{aligned}
$$

where $C_{D}=\sup _{x \in \bar{D}}\left|D^{2} u(x)\right| \leq\|u\|_{C^{2, \alpha}(\bar{D})}$.

For simplicity, denote by $g=\mathcal{M} u$ and $g^{\varepsilon}$ its mollification. For any $x_{0} \in \mathcal{O}$, suppose $B_{\theta}\left(x_{0}\right) \subset \mathcal{O}$, then for any $\varepsilon \in\left(0, \frac{\theta}{2}\right), \rho \in\left(0, \rho_{0} \wedge \frac{\theta}{2}\right)$, and a unit vector $\chi \in \mathbb{R}^{n}$,

$$
\begin{aligned}
& \frac{1}{\rho^{2}}\left[g^{\varepsilon}\left(x_{0}+\rho \chi\right)+g^{\varepsilon}\left(x_{0}-\rho \chi\right)-2 g^{\varepsilon}\left(x_{0}\right)\right] \\
= & \frac{1}{\rho^{2}} \int_{B_{\varepsilon}(0)}\left[g\left(x_{0}-z+\rho \chi\right)+g\left(x_{0}-z-\rho \chi\right)-2 g\left(x_{0}-z\right)\right] \eta^{\varepsilon}(z) d z \\
\leq & C_{D} \int_{B_{\varepsilon}(0)} \eta^{\varepsilon}(z) d z=C_{D} .
\end{aligned}
$$

Sending $\rho \rightarrow 0$ we get

$$
\chi^{\top} D^{2} g^{\varepsilon}\left(x_{0}\right) \chi \leq C_{D}
$$

Hence,

$$
\begin{aligned}
\operatorname{tr}\left(\sigma\left(x_{0}\right) \sigma\left(x_{0}\right)^{\top} D^{2} g^{\varepsilon}\left(x_{0}\right)\right) & =\operatorname{tr}\left(\sigma^{\top}\left(x_{0}\right) D^{2} g^{\varepsilon}\left(x_{0}\right) \sigma\left(x_{0}\right)\right) \\
& =\sum_{k} \sigma_{k}^{\top} D^{2} g^{\varepsilon} \sigma_{k} \\
& \leq C_{D} \sum_{i, j}\left|\sigma_{i j}\left(x_{0}\right)\right|^{2} \\
& \leq C
\end{aligned}
$$

where $\sigma_{k}$ is the $k$-th column of the matrix $\sigma, \sigma_{i j}$ is the $(i, j)$-th element of $\sigma$, and the last inequality is due to continuity of $\sigma$. 
Note that $\left|g^{\varepsilon}\left(x_{0}\right)\right|+\left|D g^{\varepsilon}\left(x_{0}\right)\right| \leq\|g\|_{W^{1, \infty}(\mathcal{O})}$ and $\mu(x)$ bounded, we deduce

$$
L g^{\varepsilon}\left(x_{0}\right)=-\frac{1}{2} \operatorname{tr}\left(\sigma\left(x_{0}\right) \sigma\left(x_{0}\right)^{\top} D^{2} g^{\varepsilon}\left(x_{0}\right)\right)-\mu\left(x_{0}\right) \cdot D g^{\varepsilon}\left(x_{0}\right)+r g^{\varepsilon}\left(x_{0}\right) \geq-M,
$$

where the constant $M$ is independent of $x_{0}$.

Finally, recall that $u$ is a viscosity of (4.3) by Corollary 4.5. We can apply Corollary 6.2 with $f$ replaced by $\tilde{f}=f+I u \in C\left(\mathbb{R}^{n}\right)$ and $g=\mathcal{M} u$ and conclude that for any $1 \leq p<\infty$,

$$
u \in W^{2, p}(\mathcal{O})
$$

\section{REFERENCES}

[1] G. Barles and C. Imbert. Second-order elliptic integro-differential equations: viscosity solutions' theory revisited. Ann. Inst. H. Poincaré Anal. Non Linéaire, 25(3):567-585, 2008.

[2] E. Bayraktar and H. Xing. Analysis of the optimal exercise boundary of american options for jump diffusions. SIAM Journal on Mathematical Analysis, 41(2):825-860, 2009.

[3] A. Bensoussan and J.-L. Lions. Impulse Control and Quasivariational Inequalities. Bordas, 1982. Translation of Contrôle Impulsionnel et Inéquations Quasi-variationnelles.

[4] T. Bielecki and S. Pliska. Risk sensitive asset management with fixed transaction costs. Finance and Stochastics, 4:1-33, 2000.

[5] J.-M. Bony. Principe du maximum dans les espaces de Sobolev. C. R. Acad. Sci. Paris Sér. A-B, 265:333-336, 1967.

[6] B. Bruder and H. Pham. Impulse control problems on finite horizon with execution delay. Stochastic Processes and their Applications, 119:1436-1469, 2009.

[7] A. Cadenillas, T. Choulli, M. Taksar, and L. Zhang. Classical and impulse stochastic control for the optimization of the dividend and risk policies of an insurance firm. Mathematical Finance, 16(1):181-202, 2006.

[8] A. Cadenillas and F. Zapatero. Optimal central bank intervention in the foreign exchange market. Journal of Econ. Theory, 97:218-242, 1999.

[9] P. Carr, H. Geman, D. Madan, and M. Yor. Stochastic volatility for Lévy processes. Mathematical Finance, 13(3):345-382, 2003.

[10] G. M. Constantinides and S. F. Richard. Existence of optimal simple policies for discounted-cost inventory and cash management in continuous time. Oper. Res., 26(4):620-636, 1978.

[11] R. Cont and P. Tankov. Financial Modelling with Jump Processes. Chapman \& Hall/CRC, Boca Raton, FL, 2004.

[12] M. G. Crandall. Viscosity solutions: a primer. In Viscosity solutions and applications (Montecatini Terme, 1995), volume 1660 of Lecture Notes in Math., pages 1-43. Springer, Berlin, 1997.

[13] M. G. Crandall, H. Ishii, and P. L. Lions. User's guide to viscosity solutions of second order partial differential equations. Bull. Amer. Math. Soc., 27:1-62, 1992.

[14] J. E. Eastham and K. J. Hastings. Optimal impulse control of portfolios. Math. Oper. Res., 13(4):588-605, 1988.

[15] E. Eberlein. Application of generalized hyperbolic Lévy motions to finance. in Lévy Processes, O. E. Barndorff-Nielsen ed., Birkhauser, Boston, MA, pages 319-336, 2001.

[16] M. G. Garroni and J. L. Menaldi. Second order elliptic integro-differential problems. Chapman \& Hall/CRC, 2002.

[17] I. I. Gihman and A. V. Skorohod. Controlled Stochastic Processes. Translated from the Russian by Samuel Kotz, Springer-Verlag, 1979.

[18] D. Gilbarg and N. S. Trudinger. Elliptic Partial Differential Equations of Second Order. Springer, 1998.

[19] X. Guo and P. Tomecek. Connections between singular control and optimal switching. SIAM J. on Control and Optimization, 47(1):421-443, 2008

[20] X. Guo and G. L. Wu. Smooth fit principle for impulse control of multidimensional diffusion processes. SIAM J. Control Optim., 48(2):594-617, 2009.

[21] J. M. Harrison, T. M. Sellke, and A. J. Taylor. Impulse control of Brownian Motion. Math. Oper. Res., 8(3):454-466, 1983.

[22] J. M. Harrison and M. I. Taksar. Instantaneous control of Brownian Motion. Math. Oper. Res., 8(3):439-453, 1983 1983.

[23] N. Ikeda and W. S. Stochastic Differential Equations and Diffusion Processes. Kodansha/North Holland, 2nd edition, 1989.

[24] H. Ishii. On the equivalence of two notions of weak solutions, viscosity solutions and distribution solutions. Funkcial. Ekvac., 38:101-120, 1995.

[25] Y. Ishikawa. Optimal control problem associated with jump processes. Applied Mathematics and Optimization, 50(1):2165, 2004.

[26] M. Jeanblanc and S. Shiryayev. Optimization of the flow of dividends. Russian Math. Surveys, 50:257-277, 1995.

[27] M. Jeanblanc-Picqué. Impulse control method and exchange rate. Mathematical Finance, 3:161-177, 1993.

[28] J. Kallsen. Optimal portfolios for expontential lévy processes. Math. Methods Oper. Res., 51:357-374, 2000.

[29] R. Korn. Protfolio optimization with strictly positive transaction costs and impulse control. Finance and Stochastics, $2: 85-114,1998$ 
[30] R. Korn. Some applications of impulse control in mathematical finance. Math. Meth. Oper. Res., 50:493-518, 1999.

[31] O. A. Ladyzhenskaya and N. N. Ural'tseva. Linear and quasilinear elliptic equations. Translated from the Russian by Scripta Technica, Inc. Translation editor: Leon Ehrenpreis. Academic Press, New York, 1968.

[32] D. Lamberton and M. Mikou. The critical price for the American put in an exponential Lévy model. Finance and Stochastics, 12(4):561-581, 2008.

[33] G. M. Lieberman. Second order parabolic differential equations. World Scientific Publishing Co. Inc., River Edge, NJ, 1996.

[34] P.-L. Lions. A remark on Bony maximum principle. Proc. Amer. Math. Soc., 88(3):503-508, 1983.

[35] V. LyVath, M. Mnif, and H. Pham. A model of optimal portfolio selection under liquidity risk and price impact. Finance and Stochastics, (1):51-90, Jan 2007.

[36] J. Ma. On the principle of smooth fit for a class of singular stochastic control problems for diffusions. SIAM J. Control Optim., 30(4):975-999, 1992.

[37] D. C. Mauer and A. Triantis. Interactions of corporate financing and investment decisions: a dynamic framework. Journal of Finance, 49(4):1253-1277, 1994.

[38] A. J. Morton and S. Pliska. Optimal portfolio management with fixed transaction costs. Mathematical Finance, 5:337-356, 1995.

[39] G. Mundaca and B. Øksendal. Optimal stochastic intervention control with application to the exchange rate. J. of Mathematical Economics, 29:225-243, 1998.

[40] B. Øksendal and A. Sulem. Optimal consumption and portfolio with both fixed and proportional transaction costs. SIAM J. Cont. Optim., 40:1765-1790, 2002.

[41] B. Øksendal and A. Sulem. Applied Stochastic Control of Jump Diffusions. Universitext. Springer-Verlag, Berlin, 2004.

[42] H. Pham. Optimal stopping, free boundary, and american option in a jump-diffusion model. Applied Mathematics and Optimization, 35(2):145-164, 1997.

[43] H. Pham. On the smooth-fit property for one-dimensional optimal switching problem. Séminaire de Probabilités, XL:187$201,2007$.

[44] R. Seydel. Existence and uniqueness of viscosity solutions for QVI associated with impulse control of jump diffusions. Stochastic Processes and their Applications, pages 3719-3748, 2009.

[45] R. C. Seydel. General existence and uniqueness of viscosity solutions for impulse control of jump-diffusions. MPI MIS Preprint 37/2008, 2008.

[46] H. M. Soner. Optimal control with state-space constraint. II. SIAM J. Control Optim., 24(6):1110-1122, 1986.

[47] M. I. Taksar. Average optimal singular control and a related stopping problem. Math. Oper. Res., 10:63-81, 1985 1985.

[48] S. J. Tang and J. M. Yong. Finite horizon stochastic optimal switching and impulse controls with a viscosity solution approach. Stochastics Stochastics Rep., 45(3-4):145-176, 1993.

[49] A. Triantis and J. E. Hodder. Valuing flexibility as a complex option. Journal of Finance, 45(2):549-565, 1990.

[50] C. Yang, L. Jiang, and B. Bian. Free boundary and american options in a jump-diffusion model. European Journal of Applied Mathematics, 17(1):95-127, 2006.

[51] J. Yong and X. Zhou. Stochastic Controls: Hamiltonian Systems and HJB Equations. Springer, 1999.

Appendix A. Proof of Theorem 6.1. A standard technique to get regularity is to consider a sequence of penalized problems. For this, let $\beta_{\varepsilon}$ denote a sequence of smooth functions satisfying

$$
\left\{\begin{array}{l}
\beta_{\varepsilon}(t) \rightarrow \infty, \text { as } \varepsilon \rightarrow 0, t>0 \\
\beta_{\varepsilon}(t) \rightarrow 0, \text { as } \varepsilon \rightarrow 0, t \leq 0 \\
0<\beta_{\varepsilon}{ }^{\prime}(t)<\omega(\varepsilon)^{-1}, \forall t \\
\beta_{\varepsilon}(0)=0, \beta_{\varepsilon} \geq-1
\end{array}\right.
$$

Here $\omega(\cdot)$ is the modulus of continuity for the convergence $g^{\varepsilon} \rightarrow g$, i.e.,

$$
\omega(\delta):=\sup _{\varepsilon \leq \delta}\left\|g^{\varepsilon}-g\right\|_{C(\overline{\mathcal{O}})} .
$$

Thus, $\omega(\varepsilon) \rightarrow 0$ as $\varepsilon \rightarrow 0$. For a given $\varepsilon>0$, the graph of $\beta_{\varepsilon}$ is shown in Figure A.1

We approximate the Dirichlet problem (6.5) with the following penalizing problems:

$$
\begin{cases}L v^{\varepsilon}+\beta_{\varepsilon}\left(v^{\varepsilon}-g^{\varepsilon}\right)=f & \text { in } \mathcal{O}, \\ v^{\varepsilon}=0 & \text { on } \partial \mathcal{O} .\end{cases}
$$

By a standard fixed point argument, (A.2) has a unique solution in $W^{2, p}(\mathcal{O}) \cap W_{0}^{1, p}(\mathcal{O})$, for any $1 \leq p<\infty$. Moreover, we have the following estimates:

Lemma A.1. Under the same assumptions as Theorem 6.1, there exists a constant $C$ independent of $\varepsilon$, such that

$$
\left\|v^{\varepsilon}\right\|_{W^{2, p}(\mathcal{O})} \leq C .
$$




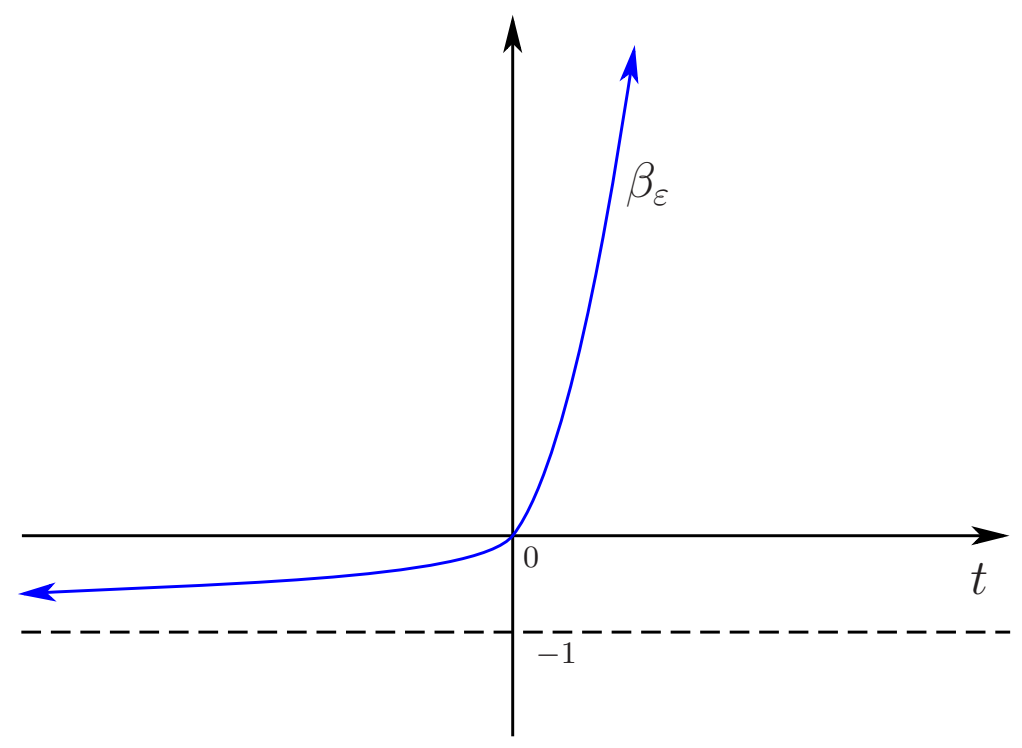

FIG. A.1. Penalizing Functions

Proof. The goal is to show that

$$
\left\|\beta_{\varepsilon}\left(v^{\varepsilon}-g^{\varepsilon}\right)\right\|_{L^{\infty}(\mathcal{O})} \leq C .
$$

Clearly, to get (A.3), it suffices to show

$$
\beta_{\varepsilon}\left(v^{\varepsilon}-g^{\varepsilon}\right) \leq C,
$$

since $\beta_{\varepsilon} \geq-1$ by construction.

Consider the point $x_{0}$ at which the maximum of $\beta_{\varepsilon}\left(v^{\varepsilon}-g^{\varepsilon}\right)$ occurs.

Case 1. If $x_{0} \in \partial \mathcal{O}$, then $v^{\varepsilon}\left(x_{0}\right)=0 \leq g\left(x_{0}\right)$. Thus

$$
v^{\varepsilon}\left(x_{0}\right)-g^{\varepsilon}\left(x_{0}\right) \leq g\left(x_{0}\right)-g^{\varepsilon}\left(x_{0}\right) \leq \omega(\varepsilon) .
$$

Because $0<\beta_{\varepsilon}{ }^{\prime}<\omega(\varepsilon)^{-1}$ and $\beta_{\varepsilon}(0)=0$, we have $\beta_{\varepsilon}\left(v^{\varepsilon}-g^{\varepsilon}\right) \leq 0$, if $v^{\varepsilon}-g^{\varepsilon} \leq 0$; or $0 \leq \beta_{\varepsilon}\left(v^{\varepsilon}-g^{\varepsilon}\right) \leq 1$ if $v^{\varepsilon}-g^{\varepsilon} \geq 0$. In either case, we have $\beta_{\varepsilon}\left(v^{\varepsilon}-g^{\varepsilon}\right) \leq 1$ at $x_{0}$.

Case 2. If $x_{0} \in \mathcal{O}$, then $v^{\varepsilon}-g^{\varepsilon}$ also attains its maximum there, since $\beta_{\varepsilon}{ }^{\prime}>0$. By Bony's maximum principle (see [5] or [34]),

$$
\text { ess } \lim _{x \rightarrow x_{0}} \sup \left\{-a_{i j}\left(v^{\varepsilon}-g^{\varepsilon}\right)_{x_{i} x_{j}}\right\} \geq 0 .
$$

Thus, either $v^{\varepsilon}-g^{\varepsilon} \leq 0$ at $x_{0}$, which implies $\beta_{\varepsilon}\left(v^{\varepsilon}-g^{\varepsilon}\right) \leq 0$, or,

$$
\text { ess } \lim _{x \rightarrow x_{0}} \sup L v^{\varepsilon} \geq L g^{\varepsilon}\left(x_{0}\right) \geq-M,
$$

due to (6.4). By continuity,

$$
\beta_{\varepsilon}\left(v^{\varepsilon}-g^{\varepsilon}\right)\left(x_{0}\right)=f\left(x_{0}\right)-\text { ess } \lim _{x \rightarrow x_{0}} \sup L v^{\varepsilon} \leq f\left(x_{0}\right)+M \leq C .
$$

Combing these two cases, we obtain (A.4). Thus, we proved (A.3), and from the PDE (A.2) we have $\left\|L v^{\varepsilon}\right\|_{L^{\infty}(\mathcal{O})} \leq C$ independently of $\varepsilon$.

Finally, the Calderon-Zygmund estimate implies the desired result. 
Thanks to Lemma A.1 we can extract a subsequence of $v^{\varepsilon}$, denoted by $v^{\varepsilon_{k}}$, such that

$$
\left\{\begin{array}{l}
v^{\varepsilon_{k}} \rightarrow \bar{v} \text { weakly in } W^{2, p}(\mathcal{O}) \\
v^{\varepsilon_{k}} \rightarrow \bar{v} \text { uniformly in } \mathcal{O}
\end{array}\right.
$$

Due to the stability result of viscosity solutions (see, for instance, [12, Theorem 8.3]), this limit function $\bar{v}$ is in fact a viscosity solution of 6.5.

Lemma A.2. The limit function $\bar{v}$ is a viscosity solution of (6.5).

Proof. (Subsolution) Suppose $\phi(x)$ is a smooth test function and $\bar{v}-\phi$ has a local maximum at $x_{0} \in \mathcal{O}$ with $\bar{v}\left(x_{0}\right)=\phi\left(x_{0}\right)$. We want to show that

$$
\max \{L \phi-f, \phi-g\} \leq 0 \text { at } x_{0} .
$$

Without loss of generality, we may assume $x_{0}$ is a strict local maximum because otherwise we can replace $\phi(x)$ by $\phi(x)-\left|x-x_{0}\right|^{4}$ and prove the same result. In this case, for any open ball with radius $\delta>0$ and centered at $x_{0}$, denoted by $B_{\delta}\left(x_{0}\right), v^{\varepsilon_{k}}-\phi$ has a local maximum $x_{k} \in B_{\delta}\left(x_{0}\right)$ for $k$ sufficiently large, because $v^{\varepsilon_{k}}$ converges to $v$ uniformly in $B_{\delta}\left(x_{0}\right)$ and $\bar{v}\left(x_{0}\right)-\phi\left(x_{0}\right)>\max _{\partial B_{\delta}\left(x_{0}\right)}(\bar{v}-\phi)$. Let $\delta$ go to zero, and we obtain

$$
x_{k} \text { is a local maximum of } v^{\varepsilon_{k}}-\phi, \quad \text { and } \lim _{k \rightarrow \infty} x_{k}=x_{0} \text {. }
$$

By the maximum principle, ess $\lim _{x \rightarrow x_{k}} \sup \left\{-a_{i j}\left(v^{\varepsilon_{k}}-\phi\right)_{x_{i} x_{j}}\right\} \geq 0$ at $x_{k}$. However, $v^{\varepsilon_{k}}$ satisfies (A.2), thus,

$$
\begin{aligned}
L \phi\left(x_{k}\right) & \leq \operatorname{ess} \lim _{x \rightarrow x_{k}} \sup L v^{\varepsilon_{k}}-r\left(v^{\varepsilon_{k}}\left(x_{k}\right)-\phi\left(x_{k}\right)\right) \\
& =f\left(x_{k}\right)-\beta_{\varepsilon_{k}}\left(v^{\varepsilon_{k}}-g^{\varepsilon_{k}}\right)\left(x_{k}\right)-r\left(v^{\varepsilon_{k}}\left(x_{k}\right)-\phi\left(x_{k}\right)\right) .
\end{aligned}
$$

Note that $v^{\varepsilon_{k}}-g^{\varepsilon_{k}} \rightarrow \bar{v}-g$ and $v^{\varepsilon_{k}}-\phi \rightarrow \bar{v}-\phi$ locally uniformly, we have

$$
\left\{\begin{array}{l}
v^{\varepsilon_{k}}\left(x_{k}\right)-g^{\varepsilon_{k}}\left(x_{k}\right) \rightarrow \bar{v}\left(x_{0}\right)-g\left(x_{0}\right), \\
v^{\varepsilon_{k}}\left(x_{k}\right)-\phi\left(x_{k}\right) \rightarrow \bar{v}\left(x_{0}\right)-\phi\left(x_{0}\right)=0,
\end{array} \quad \text { as } k \rightarrow \infty .\right.
$$

Sending $k \rightarrow \infty$ in A.5 , we obtain

$$
\phi-g=\bar{v}-g \leq 0 \text { at } x_{0} .
$$

(Otherwise, $L \phi \leq-\infty$ at $x_{0}$ since $\beta_{\varepsilon}(t) \rightarrow \infty$ as $\varepsilon \rightarrow 0$ if $t>0$ ). Moreover,

$$
L \phi \leq f \text { at } x_{0},
$$

since $\beta_{\varepsilon}(t) \rightarrow 0$ as $\varepsilon \rightarrow 0$ if $t \leq 0$. We have proved that $\bar{v}$ is a viscosity subsolution.

(Supersolution) Similarly, suppose $\phi(x)$ is a smooth test function and $\bar{v}-\phi$ has a local strict minimum at $x_{0}$ with $\bar{v}\left(x_{0}\right)=\phi\left(x_{0}\right)$. We want to show that

$$
\max \{L \phi-f, \phi-g\} \geq 0 \text { at } x_{0} .
$$

For the same reason as above, we can take a sequence $\left\{x_{k}\right\}$ so that

$$
x_{k} \text { is a local minimum of } v^{\varepsilon_{k}}-\phi, \quad x_{k} \rightarrow x_{0},
$$

and (A.6) still holds. And again by maximum principle, at $x_{k}$,

$$
L \phi \geq f-\beta_{\varepsilon_{k}}\left(v^{\varepsilon_{k}}-g^{\varepsilon_{k}}\right)-r\left(v^{\varepsilon_{k}}-\phi\right) .
$$


If $\phi-g \geq 0$ at $x_{0}$, then we have the desired inequality. Otherwise, $\phi\left(x_{0}\right)-g\left(x_{0}\right)=-2 \nu$ for some $\nu>0$. So $v^{\varepsilon_{k}}\left(x_{k}\right)-g^{\varepsilon_{k}}\left(x_{k}\right) \leq-\nu<0$ for $k$ sufficiently large by (A.6), and hence sending $k \rightarrow \infty$ in (A.7) yields

$$
L \phi \geq f \text { at } x_{0} .
$$

Thus, $\bar{v}$ is a supersolution.

Finally, the boundary condition is satisfied since $\bar{v}=0$ on $\partial \mathcal{O}$.

Proof. [Proof of Theorem6.1] Because of the above Lemma A.2 and the uniqueness of viscosity solutions for ( $(\mathrm{HJB})$, we conclude that $v=\bar{v} \in W^{2, p}(\mathcal{O})$. $\mathrm{Q}$

Proof. [Proof of Corollary 6.2.] To apply Theorem 6.1 we need to subtract from $v$ a function that has the same boundary value on $\partial \mathcal{O}$. Let $w$ be the unique solution of the Dirichlet problem

$$
\begin{cases}L w=0 & \text { in } \mathcal{O} \\ w=v & \text { on } \partial \mathcal{O}\end{cases}
$$

Then $w \in C_{\text {loc }}^{2, \alpha}(\mathcal{O}) \cap C(\overline{\mathcal{O}})$. Thus, $v_{0}:=v-w$ is a viscosity solution of

$$
\max \left\{L v_{0}-f, v_{0}-\bar{g}\right\}=0 \text { in } \mathcal{O}, \quad v_{0}=0 \text { on } \partial \mathcal{O},
$$

where $\bar{g}=g-w$. Then $\bar{g}=g-v \geq 0$ on $\partial \mathcal{O}$. Take $\bar{g}^{\varepsilon}=g^{\varepsilon}-w \in C^{2}(\mathcal{O}) \cap C(\overline{\mathcal{O}})$, satisfying $L \bar{g}^{\varepsilon}=L g^{\varepsilon} \geq-M$ in $\mathcal{O}$. All the other conditions of Theorem 6.1 are easily verified. So we have $v_{0} \in W^{2, p}(\mathcal{O})$ and $v=v_{0}+w \in$ $W_{\text {loc }}^{2, p}(\mathcal{O})$. But since $\mathcal{O} \subset \mathbb{R}^{n}$ is arbitrary, we have $v \in W^{2, p}(\mathcal{O})$ for any $\mathcal{O} \subset \mathbb{R}^{n}$. 